\title{
An Autopsy Proven Case of CSF1R-mutant Adult-onset Leukoencephalopathy with Axonal Spheroids and Pigmented Glia (ALSP) with Premature Ovarian Failure
}

\author{
Seong-Ik Kim ${ }^{1}$, Beomseok Jeon ${ }^{2}$, Jeongmo Bae ${ }^{1}$, Jae Kyung Won ${ }^{1}$, \\ Han-Joon Kim ${ }^{2}$, Jeemin Yim ${ }^{1}$, Yun Joong Kim ${ }^{3}$ and Sung-Hye Park ${ }^{1,4 *}$ \\ ${ }^{1}$ Department of Pathology, Seoul National University College of Medicine, Seoul 03080, ${ }^{2}$ Department of Neurology, Seoul \\ National University College of Medicine, Seoul 03080, ${ }^{3}$ Department of Neurology, Hallym University Sacred Heart Hospital, \\ Anyang 14068, Institure of Neuroscience, Seoul National University College of Medicine, Seoul 03080, Korea
}

\begin{abstract}
Adult-onset leukoencephalopathy with axonal spheroids and pigmented glia (ALSP) is a progressive degenerative white matter disorder caused by mutations in the tyrosine kinase domain of the CSF1R gene. ALSP is often misdiagnosed as other diseases due to its rarity and various clinical presentations such as Parkinsonism, pyramidal signs, cognitive impairment and/or psychiatric symptoms. We describe an autopsy case of ALSP with a CSFIR mutation. A 61-year-old woman presented insidious-onset gait difficulty for 12 years since her age of 49 , and premature ovarian failure since her age of 35 . At initial hospital visit, brain magnetic resonance imaging revealed hydrocephalus. Initially, Parkinson's syndrome was diagnosed, and she was prescribed L-dopa/carbidopa because of spasticity and rigidity of extremities, which had worsened. Subsequently, severe neuropsychiatric symptoms and cognitive impairment developed and radiologically, features of leukoencephalopathy or leukodystrophy were detected. She showed a down-hill course and died, 12 years after initial diagnosis. At autopsy, the brain showed severe symmetric atrophy of bilateral white matter, paper-thin corpus callosum, thin internal capsule, and marked hydrocephalus. Microscopically, diffuse loss of white matter, relatively preserved subcortical U-fibers, and many eosinophilic bulbous neuroaxonal spheroids were noted, but there was no calcification. Pigmented glia with brown cytoplasmic pigmentation were readily found in the white matter, which were positive for Periodic acid-Schiff, p62, and CD163 stains, but almost negative for CD68. Whole-exome and Sanger sequencing revealed a CSF1R mutation (c.2539G>A, p.Glu847Lys) which was reported in prior one ALSP case. This example demonstrates that ALSP could be associated with premature ovarian failure.
\end{abstract}

Key words: Autopsy, CSF1R, Leukoencephalopathy, Neuroglia, Whole exome sequencing

Received October 15,2018, Revised January 9, 2019,

Accepted January 14,2019

* To whom correspondence should be addressed.

TEL: 82-2-740-3090, FAX: 82-2-743-5530

e-mail:shparknp@snu.ac.kr

\section{INTRODUCTION}

Recently adult onset leukoencephalopathy with axonal spheroids and pigmented glia (ALSP) was proposed as a comprehensive term encompassing hereditary diffuse leukoencephalopathy with 
spheroids (HDLS) and pigmentary orthochromatic leukodystrophy (POLD), because of similar clinical and pathological features, and presence of CSF1R gene mutations [1,2]. POLD was firstly described in a Swedish family with leukoencephalopathy as an autosomal dominant disease in 1936 [3]. Since then, many sporadic cases have been reported [4]. HDLS was reported in 17 members of a Swedish family in 1984 [5]. Even before the CSF1R mutation was discovered by whole-exome sequencing in 2012 [6], there were reports that the two diseases may be a single entity, because of the similar clinical and pathological features [7]. Indeed, familial cases of HDLS and POLD show similar neuropsychiatric symptoms (depression, behavioral change, frontal release signs, etc.) and neurological symptoms (Parkinsonism, pyramidal signs, epilepsy, ataxia, etc.), and similar pathological features such as widespread loss of myelinated nerve fibers with frontal or frontotemporal predominance [7]. Additionally, several HDLS families and POLD families demonstrated pigmented macrophages and numerous spheroids, respectively, on brain autopsy [8].

More than 120 cases of ALSP have been reported to date based on confirmation of the CSF1R mutation [9]. Five Korean cases of ALSP with CSF1R mutation have been reported, but autopsy was not carried out in these five cases [10, 11]. Diagnostic criteria [12] and staging scheme for disease progression of ALSP were established through review of autopsy cases [13,14]. However, the rarity of the disease and the variety of phenotypic presentations cause difficulties in reaching accurate diagnosis; thus, this disease is often confused with other diseases such as primary progressive multiple sclerosis, central nervous system (CNS) vasculitis, Alzheimer's disease, frontotemporal dementia, corticobasal degeneration, and atypical Parkinsonism $[7,15,16]$.

In 2014, another type of leukoencephalopathy termed as the AARS2 mutation-related leukodystrophy (AARS2-L) was reported by Dallabona et al. [17]. This disease shares several clinical and radiological features with ALSP, but is associated with premature ovarian failure (ovarioleukodystrophy) in female patients and no CSF1R mutation [17-19].

Based on genetic alterations which can be autosomal dominant or recessive, or X-linked, eleven subtypes of adult onset leukoencephalopathy have been identified so far [20]. Ikeuchi et al. [20] have summarized the driver genes for the disease subtypes, which include CSF1R, NOTCH3, LMNB1, GFAP, HTRA1, the EIF2B family, ARSA, TREM2/TYROBP, AARS2, FMR1, and ABCD1, and have described the primary cellular involvement of these 11 disease subtypes.

If a genetic study is not carried out, ante-mortem diagnosis of these diseases are difficult. Here we report an ALSP case diagnosed based on findings from an autopsy and whole-exome sequencing; the patient had a history of a highly suspicious premature ovarian failure.

\section{CASE REPORT}

The patient was a 49-year-old Korean woman who presented with gait disturbance and slurred speech that had started 5 months prior to the first hospital visit. Her medical history included complaints of depressive moods two years before the visit, for which she was prescribed antidepressant medication (nortriptyline). She was diagnosed with premature ovarian failure at the age of 35 and subsequently received hormone replacement therapy. Clinical manifestations of this patient are summarized and compared with previously reported cases in Table 1 [9].

On neurological examination in the initial visit (12 years before death), her Korean Mini-Mental State Examination (K-MMSE) score was 25/30 points which was consistent with mild cognitive impairment. Physical examination revealed ataxic gait, postural instability, and left dominant bradykinesia. Deep tendon reflexes were $3+$ in the both upper and lower extremities, and the Babinski sign was observed on the left side. Abnormal rapid alternating movement was observed on her left side on cerebellar function test.

Family history revealed that her father had symptoms of dementia and gait disturbance at the age of 80 and that her sister also showed gait disturbance. However, according to the patient, no accurate neurological diagnosis was provided.

Over time, dysarthria and dysphagia became worse, and rigidity and spasticity increased, leading to a bed-ridden state that began at around 52 years of age. At that time, the patient showed intermittent psychotic features and aggressive behavior, and complained of self-voiding difficulty. At the age of 53, generalized allodynia developed. She died at the age of 61 . She had been diagnosed with premature ovarian failure at an outside hospital at the age of 35 and had received hormone replacement therapy. However, serum hormone levels such as follicle stimulating hormone (FSH) and / or estradiol (E2) and ultrasonographic findings necessary for the diagnosis of premature ovarian failure were not available.

Medications to reduce Parkinson's disease-like signs and symptoms like L-Dopa/carbidopa were prescribed only during the first year, and were ineffective. Later, the spasticity increased and baclofen was given, which was partially effective.

Laboratory test results including electrolyte levels, and adrenal function, were within normal range. Thyroid stimuating hormone (TSH) was mildly elevated to $4.23 \mu \mathrm{IU} / \mathrm{ml}$ (normal: $0.1 \sim 4.1 \mu \mathrm{IU} /$ $\mathrm{ml}$ ) and anti-TSHR and anti-microsomal antibodies were elevated to $11.0 \%$ (normal: $0 \sim 1 \%$ ) and $659 \mathrm{U} / \mathrm{ml}$ (normal: $0 \sim 60 \mathrm{U} / \mathrm{ml}$ ). 
Table 1. Clinical manifestation and brain imaging features presented in previously reported cases [9] and our patient

\begin{tabular}{|c|c|c|}
\hline & Findings from previous report & Our case \\
\hline Age of onset for female and male patients (mean \pm SD) & $\begin{array}{l}40 \pm 10 \text { years in female } \\
47 \pm 11 \text { years in male }\end{array}$ & 49 years / female \\
\hline Disease duration $($ mean $\pm \mathrm{SD})$ & $6.8 \pm 5.4$ years & 12 years \\
\hline Mode of inheritance & $\begin{array}{l}\text { Autosomal dominant inheritance or } \\
\text { sporadic occurrence }\end{array}$ & $\begin{array}{l}\text { Not definite } \\
\text { Father: dementia and gait disturbance } \\
\text { Sister: gait disturbance }\end{array}$ \\
\hline \multicolumn{3}{|l|}{ Clinical features (prevalence, $\%$ ) } \\
\hline Cognitive impairment & $94 \%$ & $\begin{array}{l}\text { Present } \\
\text { K-MMSE; 25/30 initially }\end{array}$ \\
\hline Psychiatric symptoms & $75 \%$ & Present \\
\hline $\begin{array}{l}\text { Anxiety, depression, apathy, indifference, abulia, } \\
\text { irritability, disinhibition, distraction, etc. }\end{array}$ & & Depression $\rightarrow$ aggressive behavior \\
\hline Parkinsonism & $61 \%$ & Present \\
\hline $\begin{array}{l}\text { Resting tremor, rigidity, bradykinesia, postural } \\
\text { instability }\end{array}$ & & $\begin{array}{l}\text { Postural instability, bradykinesia } \\
\text { (left dominant }) \rightarrow \text { rigidity } \uparrow\end{array}$ \\
\hline Pyramidal signs & $57 \%$ & Present \\
\hline $\begin{array}{l}\text { Hyperreflexia, spasticity, increased tone in extremi- } \\
\text { ties, pseudobular palsy }\end{array}$ & & Hyperreflexia, spasticity \\
\hline Seizures & $32 \%$ & Present \\
\hline \multirow[t]{6}{*}{ Other clinical features } & Dysarthria $(34 \%)$ & Present \\
\hline & Dysphagia $(17 \%)$ & Present \\
\hline & Ataxia $(27 \%)$ & Present \\
\hline & Sensory disturbance (10\%) & Allodynia \\
\hline & Peripheral neuropathy (2\%) & History of premature ovarian failure \\
\hline & & Voiding difficulty \\
\hline \multicolumn{3}{|l|}{ MRI findings (prevalence, \%) } \\
\hline Bilateral white matter lesions & $69 \%$ & Present \\
\hline Thining of corpus callosum & $49 \%$ & Present \\
\hline Calcification in the white matter & $14 \%$ & Absent \\
\hline
\end{tabular}

Anti-thyroglobulin antibody was $<25 \mathrm{U} / \mathrm{ml}$ (normal: $0 \sim 60 \mathrm{U} / \mathrm{ml}$ ), however, T3, Free T4, cortisol and ACTH were within normal limits. Genetic tests for spinocerebellar ataxias (SCA1, SCA2, SCA3, $S C A 6$, and $S C A 7$ ) and cerebral autosomal dominant arteriopathy with subcortical infarcts and leukoencephalopathy (NOTCH3) were all negative. A spectrophotometric assay using peripheral blood revealed that there was no increase in arylsulfatase A enzymatic activity. Galactosylcerebrosidase enzymatic activity as assayed by liquid chromatography-tandem mass spectrometry was also in the normal range. Plasma levels of very long chain fatty acids (VLCFAs) and vitamin E were normal. Although serum ceruloplasmin level was slightly higher than the normal value (45 $\mathrm{mg} / \mathrm{dL}$, normal: $15 \sim 40 \mathrm{mg} / \mathrm{dL}$ ), it was not considered as clinically relevant. Lumbar puncture and CSF analysis revealed $92 \mathrm{mg} / \mathrm{dL}$ of protein (normal: 15 45 mg/dL), $59.1 \mathrm{mg} / \mathrm{dL}$ of albumin (normal: $10 \sim 30 \mathrm{mg} / \mathrm{dL}$ ), and an IgG level of $7.1 \mathrm{mg} / \mathrm{dL}$. The IgG index was 0.286 , but the causes of the elevation of protein and albumin were unknown.

No electrophysiologic abnormalities suggestive of peripheral neuropathy or widespread denervation were observed. Video- oculography revealed no abnormality, and both eyes had normal visual evoked potential (VEP). Magnetic resonance imaging (MRI) performed at the ages of 49 and 52, (Fig. 1 and 2), which did not include diffusion weighted images (DWIs), and the MRI performed at age 52 did not include a fluid attenuated inversion recovery (FLAIR) image. The first MRI scan was performed five months after the beginning of the gait disturbance and two years after the onset of the depressive mood, when the patient was aged approximately 47 years (Fig. 1A C), which showed relative symmetrical confluent atrophy of the white matter mainly involving the frontoparietal lobes. The T2 hyperintense white matter lesion was confluent from the subcortical area to the periventricular area. Ventricular dilatation and mild cerebral atrophy were also observed. In 2008, all the findings had progressed to the occipital lobe (Fig. 1D F). The subcortical U-fibers was preserved. Gadolinium enhancement showed no enhancing lesion.

\section{Autopsy findings}

The weight of the brain before formalin fixation was $1020 \mathrm{~g}$. Grossly, the brain showed global atrophy, which was prominent in 


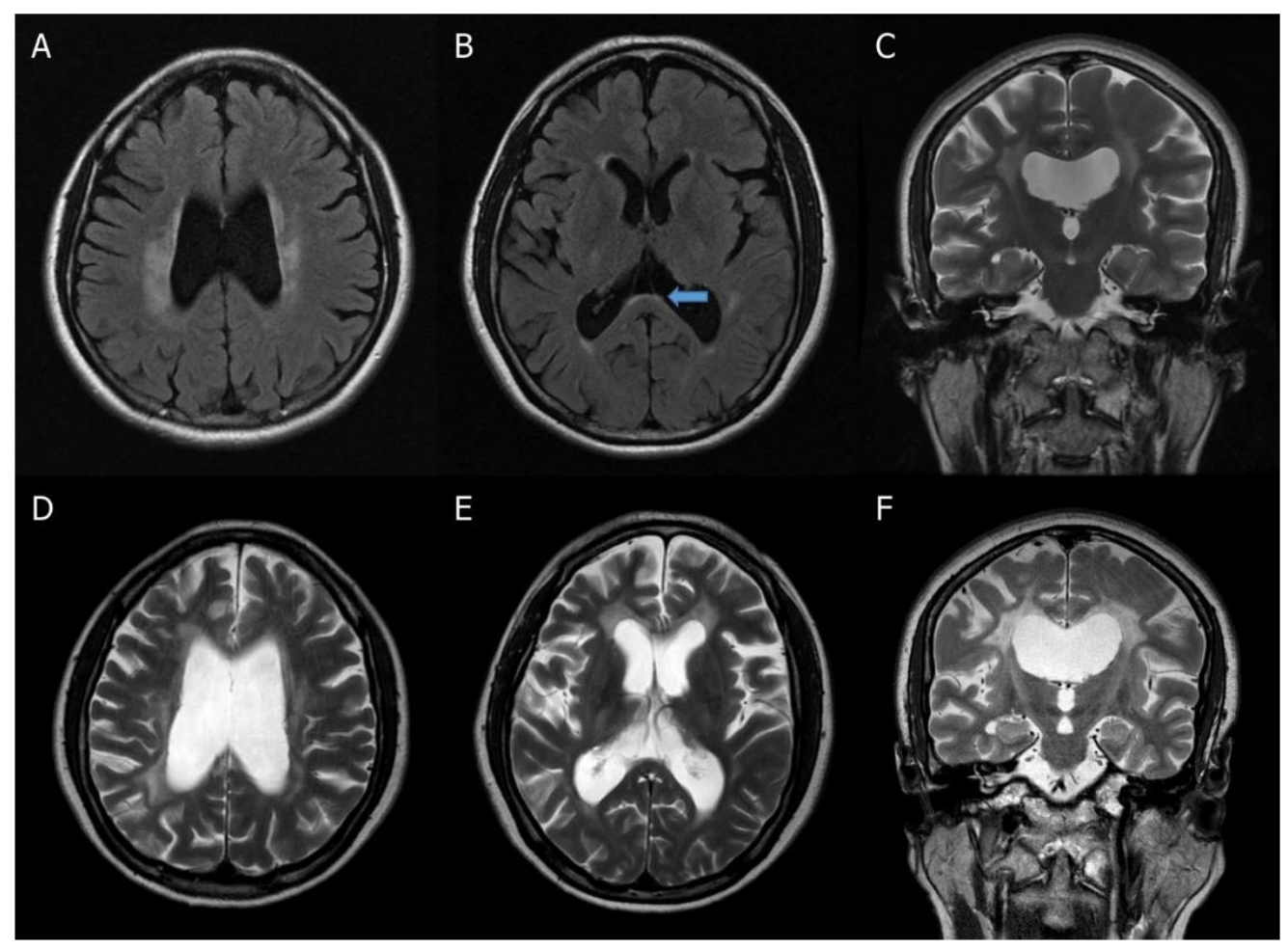

Fig. 1. Magnetic resonance imaging (MRI) findings from T2 FLAIR images (A,B) and T2-weighted image (C) obtained in 2005 and T2-weighted images obtained in 2008 (D F). There is an interval of about 3 years between $\mathrm{A} \sim \mathrm{C}$ and D F. A and D show images from almost the same level, and so are $\mathrm{B} ; \mathrm{C}$ and E;F respectively. (A C) White matter lesion with T2 hyperintensity is observed. Splenium of corpus callosum (arrow) is also involved (B). Sagittal image shows internal capsule involvement and corpus callosal thining $(\mathrm{C})$. Mild cerebral atrophy and lateral ventricular dilatation is present. (D F) In 2008, definite disease progression was detected, and the cerebral atrophy and ventricular dilatation worsened. Despite progression of the disease, the subcortical U-fibers are relatively preserved.

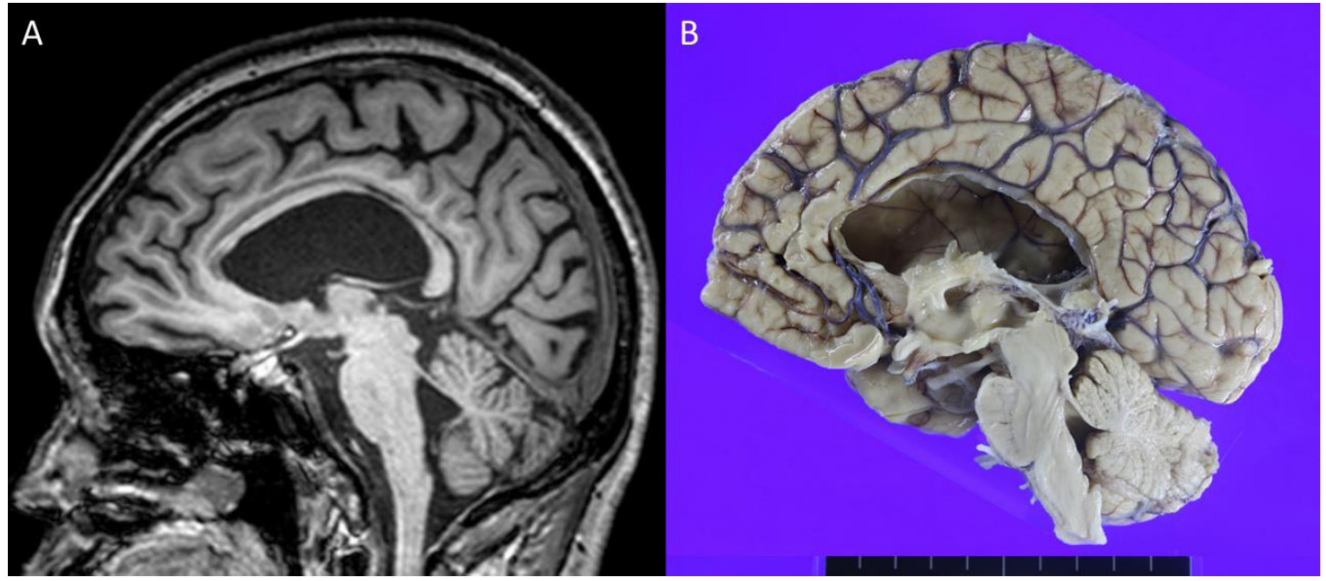

Fig. 2. (A) The Sagittal T1 image obtained in 2008 shows a thin white matter with severe hydrocephalus and atrophic cortex, cerebellum, pons, medulla and spinal cord. This section of the MRI is $0.5 \mathrm{~mm}$ lateral from the midline. (B) The formalin-fixed autopsy hemibrain obtained in 2017 shows a much thinner corpus callosum than that of MRI image obtained in 2008 and shows marked hydrocephalus. In this view, cortical atrophy does not seem severe. the frontal area. The crus cerebri and the pons were also atrophic. The corpus callosum showed a paper-thin appearance (Fig. 2B).

Through coronal section, severe atrophy of the centrum semiovale and the internal capsule with severe ventriculomegaly was detected (Fig 3A). The basal ganglia and the thalamus were also atrophic (Fig. 3A). The skin, muscle, and the ovaries were not taken during autopsy.
In order to exclude other neurodegenerative diseases, the brain tissue was stained for $\alpha$-synuclein, $\beta$-amyloid, 3 repeat (3R) tau, 4 repeat (4R) tau, p-Tau (AT8), and p-TDP43; the tissue did not yield any positive finding.

The luxol fast blue (LFB) and the myelin basic protein (MBP) staining revealed a marked loss of myelin sheath in the white matter of the frontal and parietal lobes, though the white matter of the 


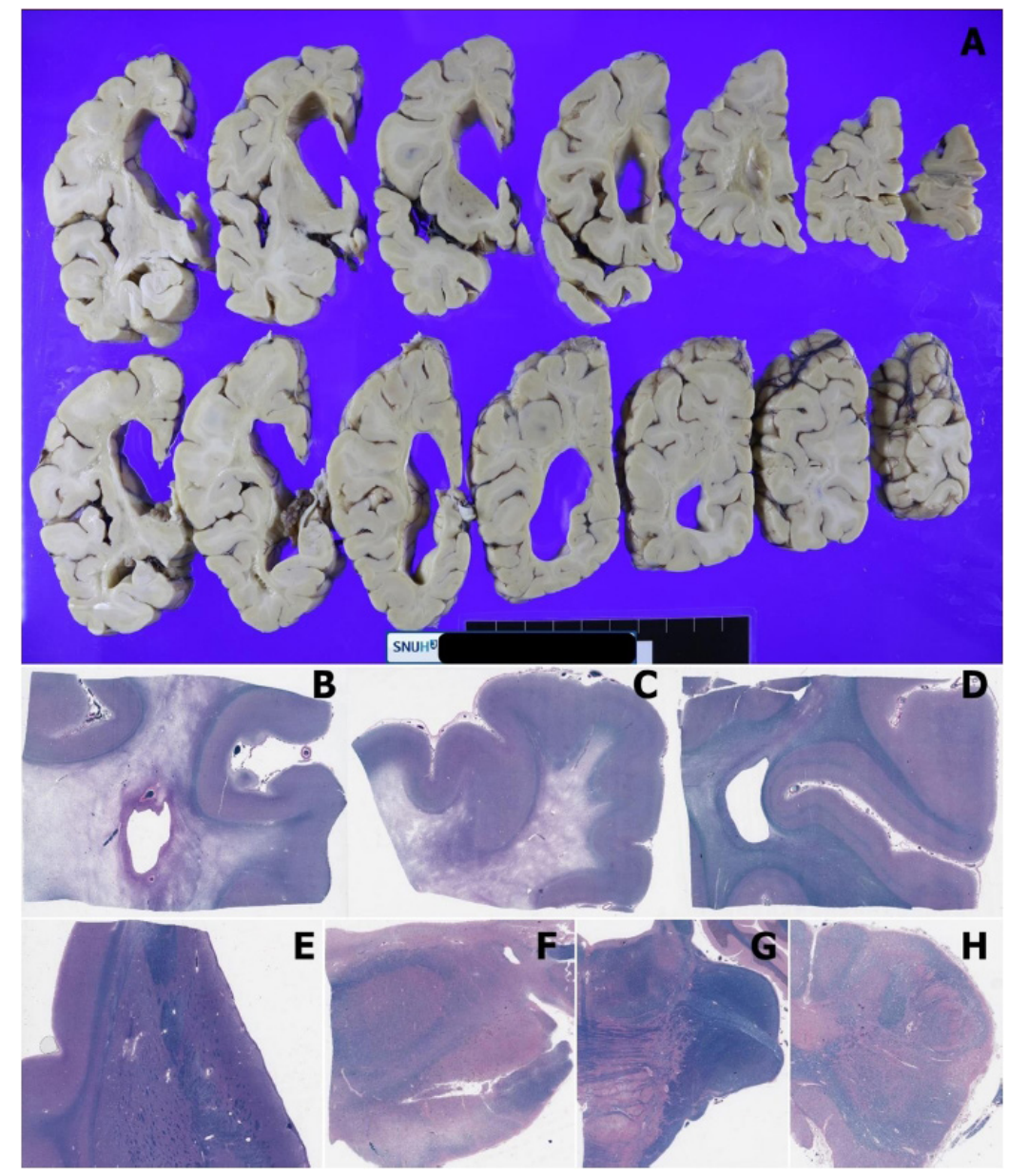

Fig. 3. Gross image from a coronal section of the cerebral hemisphere (A) and a low-power microscopic view of Luxol fast blue (LFB) staining slides from the frontal lobe (B), parietal lobe (C), occipital lobe (D), basal ganglia (E), midbrain (F), pons (G), and the cervical spinal cord (H). (A) White matter with brown-gray discoloration is observed. Corpus callosum is paper-thin and atrophied. Ventriculomegaly is evident. (B D) LFB staining reveals severe confluent myelin loss in the frontal lobe (B) and the parietal lobe $(\mathrm{C})$. The occipital lobe is relatively preserved. Subcortical U-fibers are mostly preserved. (E) The caudate nucleus, putamen, claustrum and the nigrostriatal pathway are somewhat atrophic. The anterior limb of internal capsule is relatively intact. (F H) Pyramidal tract fiber shows loss of myelin in the crus cerebri (F), basis pontis $(\mathrm{G})$, and in the anterior and lateral corticospinal tract (H). Pigmented neurons in the substantia nigra are well preserved (F).

occipital lobe was relatively preserved (Fig. 3B D). The subcortical $\mathrm{U}$-fibers are relatively preserved in the frontal and parietal lobes (Fig. 3B, C). The pyramidal tract appeared to be atrophic and demyelinated from the cerebral white matter to the cervical spinal cord (Fig. 3F H). Neurofilament (NF) and Bielschowsky silver staining revealed that most axons were lost and the remaining axons were localized in the periphery of the centrum semiovale. Neuroaxonal spheroids (Fig. 4A C) and reactive gliosis (Fig. 4I) were occasionally observed at these white matter. Neuroaxonal spheroids were also observed in parts of the neocortex including the frontal, parietal, and occipital lobes, and in the cerebellar white matter (Fig.4D and 4J). Therefore, spheroids were found in the cerebral gray and white matter, but they were more commonly seen in the gray matter. A few spheroids were observed in the midbrain and cerebellum, but not in pons, medulla oblongata and spinal cord. The maximal diameter of the axon at each location was 17.5 $\mu \mathrm{m}$ in the cerebral cortex, $31.6 \mu \mathrm{m}$ in the cerebral white matter, and $40.1 \mu \mathrm{m}$ in the cerebellar white matter.

Scattered pigmented macrophages were characteristically observed mainly in the periphery of the devastated white matter (Fig. $4 \mathrm{E})$. Occasionally, pigmented astrocyte-like cells were also observed. Positive staining for CD68 was found only in a small number of pigmented cells, but CD163 staining was robustly present in the pigmented cells (Fig. 4F, G). No calcium deposits were found in the brain. Primary antibodies used in this case were summarized in Table 2 . 


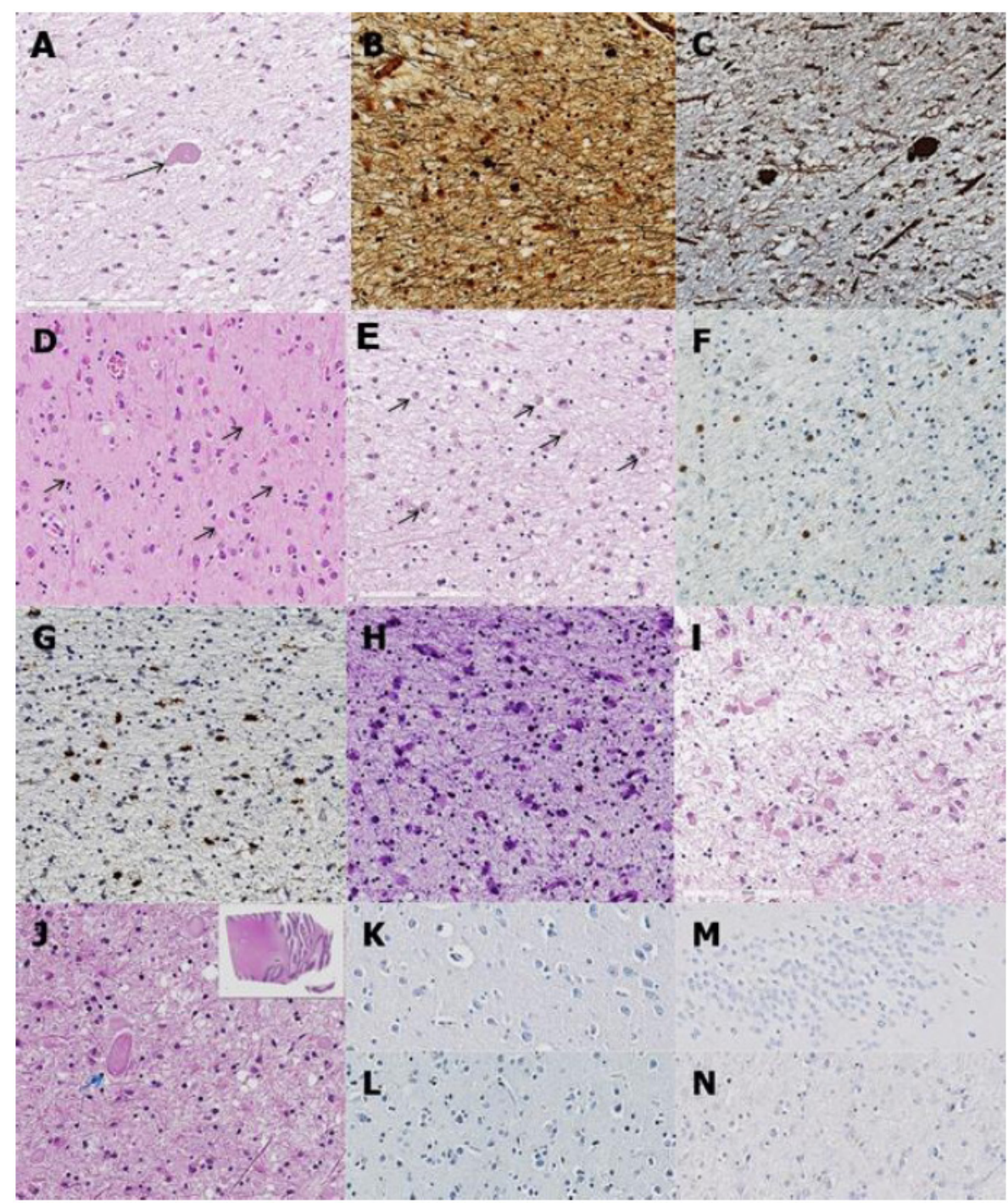

Fig. 4. High-power microscopic view of tissue stained with various stains. (A C) A spheroid (arrow) is rarely observed in the white matter of the cerebral hemisphere (A). Spheroids are emphasized via Bielschowsky silver staining (B) and neurofilament staining (C); axonal loss can also be observed with these stains. (D) The spheroids (arrows) are occasionally observed in the cortex of the parietal lobe and the density is higher than that of spheroids in the white matter. (E H) Macrophage shows hyperchromatic nuclei with brown-pigmented cytoplasm and round cellular contour (arrows) (E). CD68 immunohistochemistry shows a mostly negative status in pigmented cells (F). CD163 staining shows pigmented glial component with a ramification pattern. (G) Periodic acid-Schiff (PAS) also stained pigmented cells (H). (I) Reactive gliosis is observed in the white matter of the frontal lobe. (J) The white matter of the cerebellum is also involved and is accompanied by parenchymal vacuolation. The inset is a low-power view of the image in J., and the lesion is located in the subcortical area of the vermis. (K L) p-Tau (AT8) was negative in both hippocampal formation (K) and disease affected cerebral neocortex (L). (M N) p-TDP43 was also negative in both hippocampal formation (M) and disease affected cerebral neocortex (N). (A, D, E, I, J: Hematoxylin and eosin, B: Bielschowsky silver, C: Neurofilament, F: CD68, G: CD163, H: Periodic acid-Schiff, K, L: p-Tau (AT8) M, N: p-TDP43).

\section{Genetic study}

Genomic DNA was extracted from autopsy brain tissue using the Promega Maxwell ${ }^{\circledR}$ instrument and the PROMEGA DNA extraction kit. Samples were prepared according to the Agilent SureSelect Target Enrichment Kit preparation guide. The libraries were sequenced using the Illumina platform sequencer. The SureSelect Target Enrichment workflow is a solution-based system utilizing ultra-long 120-mer biotinylated cRNA baits to capture regions of interest, and enriching these regions of interest from a NGS genomic fragment library.

A total of $16,237,450,840$ bp were read, and the total number of reads was $160,766,840$; the GC\% was $51.2 \%$, the Q20 was $98.0 \%$, the Q30 was $94.5 \%$, the percentage of on-target reads was 79.1\%, and the mean depth of target region was 164.1. Among the Variant Call, only the variants corresponding to the patient's clinical manifestations were listed. Therefore, an annotation process was 
Table 2. Primary antibodies used in this case for diagnosis

\begin{tabular}{llll}
\hline \multicolumn{1}{c}{ Antibodies } & Dilution & \multicolumn{1}{c}{ Company } & \multicolumn{1}{c}{ Findings in this case } \\
\hline GFAP & $1: 300$ & DAKO, Glostrup, Denmark & + in reactive astrocytes \\
NeuN & $1: 500$ & Millipore, Temecula, USA & + in neurons \\
Neurofilament (NF) & $1: 2000$ & DAKO, Glostrup, Denmark & + in axons and axonal spheroids \\
Phosphorylated NF & $1: 10,000$ & Millipore, Temecula, USA & + in axons and axonal spheroids \\
Synaptophysin & $1: 100$ & Novocastra, Newcastle, UK & + in gray matter \\
CD163 & $1: 200$ & ABCAM, Bristol, UK & + in pigmented microglia \\
CD68 & $1: 2000$ & DAKO, Glostrup, Denmark & + in a few of the pigmented microglia \\
a-synuclein & $1: 200$ & ABCAM, Bristol, UK & Negative in entire brain \\
$\beta$-amyloid & $1: 500$ & Covance, Dallas, USA & Negative in entire brain \\
3 repeat (3R) tau & $1: 100$ & Millipore, Ontario, Canada & Negative in entire brain \\
4 repeat (4R) tau & $1: 1000$ & Millipore, Ontario, Canada & Negative in entire brain \\
p-Tau (AT8) & $1: 100$ & ThermoFisher, Waltham, USA & Negative in entire brain \\
p-TDP43 & $1: 1,000$ & Cosmobio, Tokyo, Japan & Negative in entire brain \\
\hline
\end{tabular}

GFAP, Glial fibrillary acidic protein; NeuN, Neuronal nuclei; CD, Cluster of differentiation; -Tau, phospho-Tau; p-TDP43, phosphorylated TAR DNA binding protein.

Table 3. List of variants found by whole exome sequencing carried out in this patient's autopsy brain tissue

\begin{tabular}{cccccccc}
\hline Gene & Mutation & Transcript ID & AA $\Delta$ & Consensus & Zygosity & Disease & Inheritance \\
\hline CSF1R & c.2539G $>$ A & ENST00000515235 & E847K & missense & het & $\begin{array}{c}\text { Leukoencephalopathy, hereditary } \\
\text { diffuse, with spheroids; ALSP }\end{array}$ \\
\hline
\end{tabular}

$\mathrm{AD}$, autosomal dominant.

performed on the selected variants. Combined Annotation Dependent Depletion (CADD), dbSNP, gnomAD, SIFT, PolyPhen, and ClinVar were used for performing the annotation. The report obtained information on CADD (Phred-like score) of 15 or more, Novel Variant or Minor Allele Frequency of less than 1\%, and VUS (Variant of Uncertain Significance) results; an interpretation of the results was carried out. The variant calling of the genes considered to be related to the patient's symptoms is shown in the Table 3.

Whole-exome sequencing using brain tissue obtained during autopsy revealed a CSF1R mutation, which was confirmed by Sanger sequencing (Fig. 5). A heterozygous missense mutation in exon 19 of the CSF1R gene (c.2539G $>$ A) was found (Table 3); this mutation, which results in the substitution of glutamic acid with lysine (p.E847K), was previously reported by Di Donato et al. [21].

\section{DISCUSSION}

ALSP is a subtype of a rare autosomal dominant, inherited leukoencephalopathy caused by a mutation in CSF1R that progressively involves white matter in the adult CNS [2]. Diseases previously diagnosed with HDLS and POLD are known to have the same genetic mutation. Therefore, ALSP is defined to include HDLS and POLD [7]. This entity may manifest as Parkinsonism and cognitive impairment. In present case, clinical manifestations started with gait disturbance and bradykinesia followed by cognitive decline, psychiatric deterioration, and severe motor impairment as summarized in Table 1.

There are eleven subtypes of adult onset leukoencephalopathy defined so far, based on genetic alterations which may be autosomal dominant or recessive or X-linked [20]. The detailed features of the eleven subtypes with their driver genes, and primary cell involvement have been summarized by Ikeuchi et al. as follows: ALSP-CSF1R-microglias, cerebral autosomal dominant arteriopathy with subcortical infarcts and leukoencephalopathy (CADASIL)-NOTCH3-small vessels, adult-onset autosomal dominant leukodystrophy (ADLD)-LMNB1-oligodendrocytes, Alexander disease-GFAP-astrocytes, cerebral autosomal recessive arteriopathy with subcortical infarcts and leukoencephalopathy (CARASIL)-HTRA1-small vessels, vanishing white matter (VWM)-EIF2B family-astrocytes, metachromatic leukodystrophy (MLD)-ARSA-oligodendrocytes, Nasu-Hakola disease-TREM2/ $T Y R O B P$-microglias, $A A R S 2$ mutation-related leukodystropy (AARS2-L)-AARS2-mitochondria, fragile X-associated tremor and ataxia syndrome (FXTAS)-FMR1-unknown, and adrenoleukodystrophy (ALD)- $A B C D 1$-oligodendrocytes [20].

Although the predominant pathology of each subtype has been reported, it may be difficult to accurately identify subtypes unless we identify the genetic variation through DNA sequencing. Each 


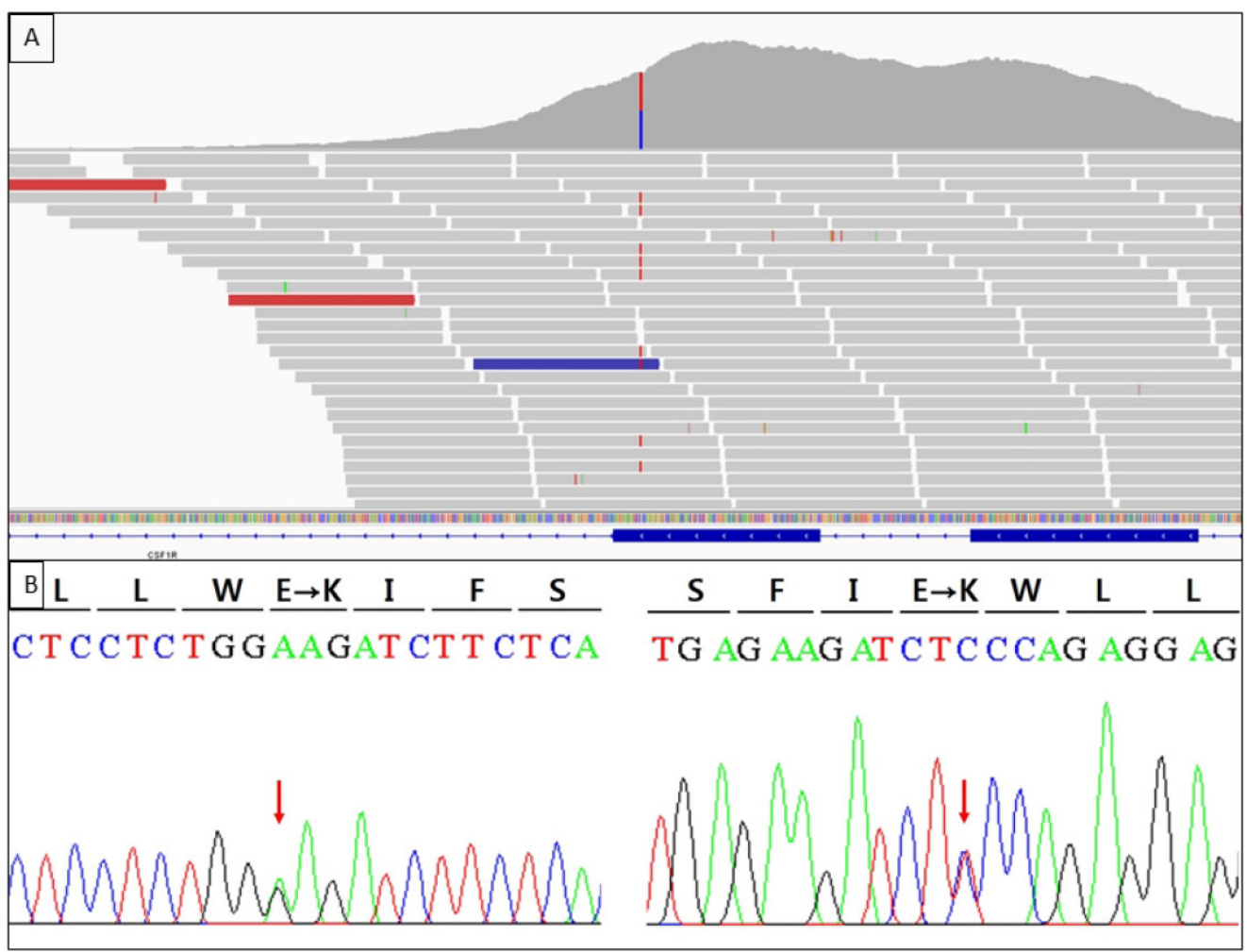

Fig. 5. Heterozygous c.2539G> A missense mutation of the CSF1R gene. (A) An integrative genomic viewer (IGV) display of a BAM (binary alignment/map) file in the CSF1R gene region. Coverage plot and aligned short reads mapped to the reference sequence are seen. (B) Sequence chromatogram of the CSF1R exon 19 in forward (left) and reverse (right) direction. This is a highly conserved region. $\mathrm{Mu}-$ tated nucleotide is indicated by an arrow. subtype exhibits significant heterogeneity and there is a significant overlap of clinical, radiological and pathological features among the subtypes. For example, ALSP shares pathological features with Nasu-Hakola disease and the primary pathology of these two entities is microglias [22,23]. ALSP and Nasu-Hakola disease characteristically represent pigmented microglia in addition to leukoencephalopathy and axonal spheroids. Nasu-Hakola disease is an autosomal recessive disorder and show relatively well preserved internal capsule and pontine base (crus cerebri), distinct from ALSP [13]. ALSP also shares clinical and radiological features with AARS2-L [17, 18]. Although some authors suggest that ALSP and AARS2-L are similar in histopathology [19], the histopathology of AARS2-L was not fully described because of the lack of autopsyproven AARS2-L.

AARS2-L usually presents with childhood- to adulthood-onset neurological deterioration such as ataxia, spasticity, cognitive decline, and frontal lobe dysfunction, which are common findings in ALSP [17]. The previous reports have emphasized premature ovarian failure in female patients, periventricular white matter rarefaction with suppression of the FLAIR signal, and absence of periventricular calcification, an important features of AARS2, distinct from ALSP $[18,19]$. However, these findings are not entirely exclusive to AARS2.

The ALSP case we report here included a history of premature ovarian failure. The most common causes of amenorrhea in women after normal secondary sexual characteristics and normal pelvic anatomy are polycystic ovarian syndrome, hyperprolactinemia, primary ovarian insufficiency, and hypothalamic dysfunction [24]. Overt primary ovarian insufficiency is defined as the presence of amenorrhea for more than 4 months with a menopausal serum FSH levels for a woman who is less than 40 years of age [24]. Thus, if women with normal secondary sexual characteristics present with amenorrhea, assessment of serum prolactin, thyroid stimulating hormone (TSH) levels, and FSH levels is helpful for accurate diagnosis. However, these hormone levels and sonographic finding of the ovary at the time of diagnosis of premature ovarian failure could not be obtained from this patient. Therefore we could not concluded the etiology of ovarian failure. However, considering the fact that the patient had received hormone replacement therapy and that there was no history of long-term hospital visits or remedies for other symptoms related to the above listed abnormalities during the following period, the possibility of premature ovarian failure is high.

Ovarioleukodystrophy, which is defined as the co-occurrence of leukodystrophy and premature ovarian failure, is a genetically heterogenous syndrome; to date, VWM and AARS2-L have been linked to premature ovarian failure $[25,26]$. Therefore, if leukodystrophy is suspected in the patients with premature ovarian fail- 
ure, genetic testing should cover evaluation of EIF2B family and AARS2. Our patient showed wildtype EIF2B family and AARS2.

Although there is a report that POLD can be linked to premature ovarian failure [27], as far as we know, an association between premature ovarian failure and genetically confirmed ALSP has not been previously reported.

Another unique feature of this case is the allodynia, a common feature of the neuropathic pain, which is abnormal perception of pain by non-painful mechanical or thermal stimuli [28]. The allodynia can be generalized or focal. Cord injury induced pain may involve the diffuse body region below the level of injury [29]. Peripheral neuropathy can also induce allodynia in the distal part of the limbs. However, the generalized pattern of this patient's allodynia suggests central origin, which is also supported by normal electromyography (EMG) and nerve conduction study (NCS). Allodynia is usually not a symptom of leukoencephalopathy, however, it is rarely reported in the patients with leukoencephalopathy, including ALSP [30,31]. Therefore, central origin allodynia is suggested in this patient, but fibromyalgia or somatic allodynia cannot be ruled out.

Leukoencephalopathy can involve any part of the white matter and may manifest with a variety of symptoms. From the viewpoint of clinico-pathologic correlation, a depressive mood since the age of 47 may be related to ALSP. The extrapyramidal symptoms exhibited by the patient may have been related to the pathology of the basal ganglia, because this region is atrophic. Ataxia is can be associated with cerebellar lesions of ALSP, but can also be caused by factors other than cerebellar lesions [7].

Several previous studies have reported on the temporal and spatial sequence of ALSP [13,32]. Our patient's disease duration was about 12 years, with severe white matter loss extending to the occipital lobe, dominance of CD163-positive over CD68-positive microglial subsets, and involvement of the cerebellum and the spinal cord; these features suggest late-stage disease, as compared to that reported in previous studies (Supplementary Table 1).

A previous study reported that calcifications are known to be present in the white matter in about 3 54\% of ALSP cases [9, 33], however our case did not show any calcification.

Over 60 mutation foci of the CSF1R gene have been identified in ALSP [1]. The mutations in exon 19 of the CSF1R gene (c.2539G>A, p.Glu847Lys) found in our patient have been reported in one previous case of ALSP as a novel mutation [21]. Di Donato et al. [21] reported on an ALSP case in which peripheral neuropathy on electrophysiological examination and parietooccipital predominant nature were apparent, but these features were not observed in our case.

Here we report a case of CSF1R gene-mutant and autopsy- proven ALSP with a history of premature ovarian failure. Autopsy findings are pathognomonic for this disease, however, due to clinicopathological heterogeneity and overlap in various other diseases including Parkinson's disease and some leukoencephalopathies, genetic study is mandatory for confirmation of diagnosis.

\section{ACKNOWLEDGEMENTS}

This research was supported by a fund (2018-ER6201-00) by Research of Korea Centers for Disease Control and Prevention.

\section{REFERENCES}

1. Adams SJ, Kirk A, Auer RN (2018) Adult-onset leukoencephalopathy with axonal spheroids and pigmented glia (ALSP): integrating the literature on hereditary diffuse leukoencephalopathy with spheroids (HDLS) and pigmentary orthochromatic leukodystrophy (POLD). J Clin Neurosci 48:42-49.

2. Sundal C, Wszolek ZK (1993) CSF1R-related adult-onset leukoencephalopathy with axonal spheroids and pigmented glia. In: GeneReviews ${ }^{\circledR}$ (Adam MP, Ardinger HH, Pagon RA, Wallace SE, Bean LJH, Stephens K, Amemiya A, eds). University of Washington, Seattle, Seattle, WA.

3. Van Bogaert L, Nyssen R (1936) Le type tardif de la leukodystrophie progressive familiale. Rev Neurol 65:21-45.

4. Seiser A, Jellinger K, Brainin M (1990) Pigmentary type of orthochromatic leukodystrophy with early onset and protracted course. Neuropediatrics 21:48-52.

5. Axelsson R, Röyttä M, Sourander P, Akesson HO, Andersen O (1984) Hereditary diffuse leucoencephalopathy with spheroids. Acta Psychiatr Scand Suppl 314:1-65.

6. Rademakers R, Baker M, Nicholson AM, Rutherford NJ, Finch N, Soto-Ortolaza A, Lash J, Wider C, Wojtas A, DeJesusHernandez M, Adamson J, Kouri N, Sundal C, Shuster EA, Aasly J, MacKenzie J, Roeber S, Kretzschmar HA, Boeve BF, Knopman DS, Petersen RC, Cairns NJ, Ghetti B, Spina S, Garbern J, Tselis AC, Uitti R, Das P, Van Gerpen JA, Meschia JF, Levy S, Broderick DF, Graff-Radford N, Ross OA, Miller BB, Swerdlow RH, Dickson DW, Wszolek ZK (2011) Mutations in the colony stimulating factor 1 receptor (CSF1R) gene cause hereditary diffuse leukoencephalopathy with spheroids. Nat Genet 44:200-205.

7. Wider C, Van Gerpen JA, DeArmond S, Shuster EA, Dickson DW, Wszolek ZK (2009) Leukoencephalopathy with spheroids (HDLS) and pigmentary leukodystrophy (POLD): a single entity? Neurology 72:1953-1959.

8. Marotti JD, Tobias S, Fratkin JD, Powers JM, Rhodes CH 
(2004) Adult onset leukodystrophy with neuroaxonal spheroids and pigmented glia: report of a family, historical perspective, and review of the literature. Acta Neuropathol 107:481488.

9. Konno T, Yoshida K, Mizuno T, Kawarai T, Tada M, Nozaki H, Ikeda SI, Nishizawa M, Onodera O, Wszolek ZK, Ikeuchi $\mathrm{T}$ (2017) Clinical and genetic characterization of adult-onset leukoencephalopathy with axonal spheroids and pigmented glia associated with CSF1R mutation. Eur J Neurol 24:37-45.

10. Kim EJ, Shin JH, Lee JH, Kim JH, Na DL, Suh YL, Hwang SJ, Lee JH, Lee YM, Shin MJ, Lee MJ, Kim SJ, Yoon U, Park DY, Jung DS, Ahn JW, Sung S, Huh GY (2015) Adult-onset leukoencephalopathy with axonal spheroids and pigmented glia linked CSF1R mutation: report of four Korean cases. J Neurol Sci 349:232-238.

11. Lee D, Yun JY, Jeong JH, Yoshida K, Nagasaki S, Ahn TB (2015) Clinical evolution, neuroimaging, and volumetric analysis of a patient with a CSF1R mutation who presented with progressive nonfluent aphasia. Parkinsonism Relat Disord 21:817-820.

12. Konno T, Yoshida K, Mizuta I, Mizuno T, Kawarai T, Tada M, Nozaki H, Ikeda SI, Onodera O, Wszolek ZK, Ikeuchi T (2018) Diagnostic criteria for adult-onset leukoencephalopathy with axonal spheroids and pigmented glia due to CSF1R mutation. Eur J Neurol 25:142-147.

13. Oyanagi K, Kinoshita M, Suzuki-Kouyama E, Inoue T, Nakahara A, Tokiwai M, Arai N, Satoh JI, Aoki N, Jinnai K, Yazawa I, Arai K, Ishihara K, Kawamura M, Ishizawa K, Hasegawa K, Yagisita S, Amano N, Yoshida K, Terada S, Yoshida M, Akiyama H, Mitsuyama Y, Ikeda SI (2017) Adult onset leukoencephalopathy with axonal spheroids and pigmented glia (ALSP) and Nasu-Hakola disease: lesion staging and dynamic changes of axons and microglial subsets. Brain Pathol 27:748769.

14. Alturkustani M, Keith J, Hazrati LN, Rademakers R, Ang LC (2015) Pathologic staging of white matter lesions in adultonset leukoencephalopathy/leukodystrophy with axonal spheroids. J Neuropathol Exp Neurol 74:233-240.

15. Lynch DS, Jaunmuktane Z, Sheerin UM, Phadke R, Brandner S, Milonas I, Dean A, Bajaj N, McNicholas N, Costello D, Cronin S, McGuigan C, Rossor M, Fox N, Murphy E, Chataway J, Houlden H (2016) Hereditary leukoencephalopathy with axonal spheroids: a spectrum of phenotypes from CNS vasculitis to parkinsonism in an adult onset leukodystrophy series. J Neurol Neurosurg Psychiatry 87:512-519.

16. Wong JC, Chow TW, Hazrati LN (2011) Adult-onset leukoencephalopathy with axonal spheroids and pigmented glia can present as frontotemporal dementia syndrome. Dement Geriatr Cogn Disord 32:150-158.

17. Dallabona C, Diodato D, Kevelam SH, Haack TB, Wong LJ, Salomons GS, Baruffini E, Melchionda L, Mariotti C, Strom TM, Meitinger T, Prokisch H, Chapman K, Colley A, Rocha H, Ounap K, Schiffmann R, Salsano E, Savoiardo M, Hamilton EM, Abbink TE, Wolf NI, Ferrero I, Lamperti C, Zeviani M, Vanderver A, Ghezzi D, van der Knaap MS (2014) Novel (ovario) leukodystrophy related to AARS2 mutations. Neurology 82:2063-2071.

18. Lakshmanan R, Adams ME, Lynch DS, Kinsella JA, Phadke R, Schott JM, Murphy E, Rohrer JD, Chataway J, Houlden H, Fox NC, Davagnanam I (2017) Redefining the phenotype of ALSP and AARS2 mutation-related leukodystrophy. Neurol Genet 3:e135.

19. Lynch DS, Zhang WJ, Lakshmanan R, Kinsella JA, Uzun GA, Karbay M, Tüfekçioglu Z, Hanagasi H, Burke G, Foulds N, Hammans SR, Bhattacharjee A, Wilson H, Adams M, Walker M, Nicoll JA, Chataway J, Fox N, Davagnanam I, Phadke R, Houlden H (2016) Analysis of mutations in AARS2 in a series of $C S F 1 R$-negative patients with adult-onset leukoencephalopathy with axonal spheroids and pigmented glia. JAMA Neurol 73:1433-1439.

20. Ikeuchi T, Mezaki N, Miura T (2018) Cognitive dysfunction and symptoms of movement disorders in adult-onset leukoencephalopathy with axonal spheroids and pigmented glia. Parkinsonism Relat Disord 46 Suppl 1:S39-S41.

21. Di Donato I, Stabile C, Bianchi S, Taglia I, Mignarri A, Salvatore S, Giorgio E, Brusco A, Simone I, Dotti MT, Federico A (2015) A novel CSF1R mutation in a patient with clinical and neuroradiological features of hereditary diffuse leukoencephalopathy with axonal spheroids. J Alzheimers Dis 47:319-322.

22. Alturkustani M, Sharma M, Hammond R, Ang LC (2013) Adult-onset leukodystrophy: review of 3 clinicopathologic phenotypes and a proposed classification. J Neuropathol Exp Neurol 72:1090-1103.

23. Sundal C, Lash J, Aasly J, Øygarden S, Roeber S, Kretzschman H, Garbern JY, Tselis A, Rademakers R, Dickson DW, Broderick D, Wszolek ZK (2012) Hereditary diffuse leukoencephalopathy with axonal spheroids (HDLS): a misdiagnosed disease entity. J Neurol Sci 314:130-137.

24. Berek JS, Novak E (2011) Berek and Novak's gynecology. 15th ed. Lippincott Williams \& Wilkins, Philadelphia, PA.

25. van der Knaap MS, Abbink TE (2018) Ovarioleukodystrophy: vanishing white matter versus $A A R S 2$-related ovarioleukodystrophy. Clin Neurol Neurosurg 171:195.

26. Ibitoye RT, Renowden SA, Faulkner HJ, Scolding NJ, Rice 
CM (2016) Ovarioleukodystrophy due to EIF2B5 mutations. Pract Neurol 16:496-499.

27. Verghese J, Weidenheim K, Malik S, Rapin I (2002) Adult onset pigmentary orthochromatic leukodystrophy with ovarian dysgenesis. Eur J Neurol 9:663-670.

28. Ropper AH, Samuels MA (2009) Adams and Victor's principles of neurology. 9th ed. McGraw-Hill Medical, New York, NY.

29. Canavero S, Bonicalzi V (2018) Central pain syndrome. 3rd ed. Springer International Publishing, Cham.

30. Joshi S, Yau W, Kermode A (2017) CADASIL mimicking multiple sclerosis: the importance of clinical and MRI red flags. J Clin Neurosci 35:75-77.

31. Coomans C, Sieben A, Lammens M, Ceuterick-de Groote
C, Vandenbroecke C, Goethals I, Van Melkebeke D, Hemelsoet D (2018) Early-onset dementia, leukoencephalopathy and brain calcifications: a clinical, imaging and pathological comparison of ALSP and PLOSL/Nasu Hakola disease. Acta Neurol Belg 118:607-615.

32. Freeman SH, Hyman BT, Sims KB, Hedley-Whyte ET, Vossough A, Frosch MP, Schmahmann JD (2009) Adult onset leukodystrophy with neuroaxonal spheroids: clinical, neuroimaging and neuropathologic observations. Brain Pathol 19:39-47.

33. Gore E, Manley A, Dees D, Appleby BS, Lerner AJ (2016) A young-onset frontal dementia with dramatic calcifications due to a novel CSF1R mutation. Neurocase 22:257-262. 